\title{
Electrochemical nano-roughening of gold microstructured electrodes for the enhanced detection of copper and glucose
}

Authors: Eduardo González-Martínez, Sokunthearath (Kevin) Saem, Nadine E. Beganovic and Jose MoranMirabal*

Department of Chemistry and Chemical Biology, McMaster University, Hamilton, Canada

\section{Abstract}

One of the main challenges for electrochemical sensor miniaturization is the fabrication of electrodes with a smaller footprint, while maintaining, or even increasing, their sensitivity for the targeted application. Our research group has previously demonstrated the enhancement of the electroactive surface area of gold electrodes up to 6-fold, relative to planar gold electrodes with the same footprint, through the generation of a wrinkled thin film surface via thermal shrinking. In this work, the electroactive surface area of wrinkled gold electrodes was further enhanced up to 5-fold (30-fold over flat electrodes) using a chronoamperometric pulsing technique. Scanning electron microscopy images showed progressive increase of surface roughness in response to an increasing number of applied pulses. The resulting nanoroughened electrodes present several advantages in addition to the enhanced electroactive surface area. These electrodes offer superior fouling resistance compared to that of wrinkled and flat electrodes when submerged in a solution containing bovine serum albumin at high concentrations. Cyclic voltammetry data also revealed greater sensitivity of nanoroughened electrodes toward anodic copper stripping, where the limit of quantification of copper by the nano-roughened electrodes was $0.3 \mathrm{ppm}$. Nano-roughened electrodes also allowed the highly sensitive enzyme-free detection of glucose through chronoamperometry, with a limit of detection of $0.5 \mathrm{mM}$, whereas planar electrodes did not demonstrate any ability to oxidize glucose. We foresee that this methodology to fabricate nanostructured electrodes will accelerate the development of simple, cost-effective and high sensitivity electrochemical platforms.

\section{Introduction}

Electrochemical sensing has rapidly evolved into one of the most versatile techniques in science, having applications in a variety of fields, including food industry, medicine, environment and agriculture.[1-7] 
However, the miniaturization of electrochemical sensors remains a challenge in modern electrochemistry, as the substantial decrease in the footprint of the working electrode often results in a reduction of its electroactive surface area (ESA) and consequently a decrease in sensitivity. Several approaches have been pursued for the fabrication of high ESA electrodes with a minimal footprint. A common method to enhance the ESA is the nano-structuring of gold surfaces through the formation of nanopillar arrays, as introduced by Schröper et al.[8] Using this approach, the ESA of the fabricated electrodes showed a 5.9fold increase relative to planar electrodes, for a kinetically-controlled process. Another method entails the deposition of gold nanoparticles; Murata et al.[9] obtained a roughening factor $>200$ by drop-casting gold nanoparticles onto a polycrystalline gold electrode. Other methods to enhance the ESA of gold electrodes include micropore generation using polymeric microparticles[10, 11] and nanofiber deposition.[12] However, the most widely used method to produce high ESA gold sensor surfaces is the production of nanoporous gold (NPG) electrodes, which can be produced through dealloying, dynamic hydrogen bubble templating, and electrochemical techniques.[13]

NPG electrodes have received much attention by virtue of their high catalytic activity. This feature has been attributed to the high-curvature features presented by the surface topography of these sensors. These regions are abundantly populated with low coordinated atoms that have the ability to interact more strongly with target molecules than the close-packed atoms in bulk gold.[14] The use of NPG electrodes for the electro-oxidation of glucose is a promising alternative for the enzyme-free detection of glucose. Chen et al.[15] produced NPG surfaces by dealloying a film made from a Au/Ag alloy in nitric acid, and determined that pore size and responsiveness toward glucose electro-oxidation of the NPGs depended on the dealloying time. Alternatively, Pei et al.[16] generated NPG through a one-step anodization strategy, from which a linear range of $2 \mu \mathrm{M}$ to $8.11 \mathrm{mM}$ for glucose detection was achieved. In addition to sensitivity, the implementation of a sensor for use with biofluids, like blood, demands overcoming the obstacle of fouling, a process by which the analyte of interest is prevented from reaching the active surface of the sensor. NPG have been reported to exhibit antifouling properties.[17-20]

Although effective, all these strategies require additional materials or produce waste by products, resulting higher fabrication costs. Our research group has recently developed a simple and inexpensive approach to fabricate microstructured electrodes (MSEs), based on vinyl stencil masking, gold sputtering and thermal shrinking of shape-memory polystyrene (PS) substrates.[21] The surface of the microstructured electrodes produced in this way presents a characteristic buckling pattern, whose topography depends on the initial thickness of the gold film. We have evaluated gold film thicknesses ranging from 2 to $400 \mathrm{~nm}$, 
an observed that all yielded 6-7-fold increases in ESA, when compared with planar electrodes of the same footprint.[21-23]. Here, we demonstrate that the ESA of gold MSEs fabricated through thermal shrinking and wrinkling can be further enhanced using a simple and cost-effective chronoamperometric pulsing technique. The resulting electrodes are cost effective and possess superb antifouling capacity and excellent responsiveness to the electro-oxidation of glucose, properties which highlight their potential for the development of highly sensitive biosensors for the detection of analytes in biofluids.

\section{Materials and Methods}

\subsection{Microstructured Electrode (MSE) Fabrication}

MSEs were produced using a vinyl stencil, sputter deposition, and thermal shrinking method previously described and shown in Figure 1.[21] In brief, polystyrene sheets (PS, Shrink Film, Graphix, Maple Heights, OH, USA) were cleaned in ethanol, isopropanol, and $18.2 \mathrm{M} \Omega \cdot \mathrm{cm}$ water baths for 5 minutes each, followed by drying under a nitrogen stream. An adhesive vinyl electrode mask was cut using a robotic craft cutter (ROBOPro CE5000-40-CRP, Graphtec America Inc., Irvine, CA, USA) and affixed onto the clean PS sheet. The masked PS sheet was then cut into individual electrode-sized substrates that were coated with gold (99.999\% purity, LTS Chemical Inc., Chestnut Ridge, NY, USA) using a Torr Compact Research Coater CRC-600 manual planar magnetron sputtering system (Torr International, New Windsor,

NY, USA) at deposition rates of $0.5 \AA / \mathrm{s}$. The vinyl mask was removed to reveal a uniform, planar gold electrode design on the PS. The planar electrodes were heated in an oven at $160{ }^{\circ} \mathrm{C}$ for 5 minutes to shrink the PS substrate and buckle the thin gold films. These electrodes were used for sensing or underwent further enhancement via electrochemical roughening.

\subsection{Electrochemical Roughening}

MSEs were roughened in an acidic solution using an electrochemical pulsing technique that dissolves and redeposits gold on the electrode surface, leading to a three-dimensional, porous topography. Each MSE was masked with a rectangular vinyl to expose only the circular working pad (Figure 1). The masked MSE was submerged into a 50 or $500 \mathrm{mM} \mathrm{H}_{2} \mathrm{SO}_{4}$ solution. Electrochemical pulsing was performed between 0 and $2 \mathrm{~V}$ for $2 \mathrm{~ms}$ each, using a CHI600E electrochemical workstation (CH Instruments, Austin, TX, USA) with a platinum auxiliary electrode and a silver/silver chloride reference electrode. The pulsing was performed for a set number of cycles, producing roughened microstructured electrodes (R-MSEs). 


\subsection{Antifouling Test}

The antifouling capabilities of the electrodes were assessed using a bovine serum albumin (BSA, SigmaAldrich, St. Louis, MO, USA) blocking assay and measuring the degree of electrochemical signal suppression. Planar, MSE and R-MSE electrodes were submerged in a BSA solution containing $10 \mathrm{mM}$ potassium ferrocyanide $\left(\mathrm{KFeCy}-\mathrm{K}_{4}\left[\mathrm{Fe}(\mathrm{CN})_{6}\right] \cdot 3 \mathrm{H}_{2} \mathrm{O}\right.$, Sigma-Aldrich, Oakville, ON, Canada); BSA solutions were prepared in $1 \mathrm{x}$ PBS (phosphate buffered saline, $\mathrm{pH}=7.4$ ) to final concentrations of 0.5 , 2.0 and $40.0 \mathrm{mg} / \mathrm{mL}$. Cyclic voltammetry $(\mathrm{CV})$ experiments between 0 and $0.4 \mathrm{~V}$ were performed to monitor electrode fouling caused by BSA adsorption onto the electrode surfaces.

\subsection{Water Contact Angle}

Water contact angle was determined using a Future Digital Scientific optical contact angle (OCA 35, Westbury, NY, USA) instrument equipped with an xyz motorized stage and camera for automatic measurements of water droplet contact angle.

\subsection{Electrochemical Sensing}

\subsubsection{Copper Anodic Stripping Voltammetry (ASV)}

To perform copper sensing in solution, anodic stripping voltammetry was carried out using a CHI600E electrochemical workstation (CH Instruments, Austin, TX, USA). A $10 \mathrm{mM}$ stock solution of copper chloride (Sigma-Aldrich, Sigma-Aldrich, St. Louis, MO, USA) was diluted in $0.1 \mathrm{mM}$ sulfuric acid to the desired working concentrations. Each gold working electrode was submerged in the copper chloride solution, following which a $-100 \mathrm{mV}$ potential was applied for 180 seconds to adsorb copper onto the working electrode surface. The copper-coated electrode was then placed in a $500 \mathrm{mM}$ sulfuric acid sensing solution in which CV or linear sweep voltammetry (LSV) was performed between 0 and $600 \mathrm{mV}$ against a platinum auxiliary electrode and a silver/silver chloride reference electrode. This procedure was repeated for each relevant copper concentration. Electrodes were cleaned and reused by performing 5 $\mathrm{CV}$ scans in $500 \mathrm{mM}$ sulfuric acid between 0.2 and $1.8 \mathrm{~V}$ or by applying a potential of $0.32 \mathrm{~V}$ for 60 seconds.

\subsubsection{Glucose Detection}

A $50 \mathrm{mM}$ glucose $(\mathrm{D}(+)$ Glucose, Sigma-Aldrich, St. Louis, MO, USA) stock solution was prepared in 1x PBS. Dilution of the glucose stock solution to the desired working concentrations was carried out in 1x PBS. A diluted glucose solution was pipetted into an electrochemical cell and chronoamperometry 
was performed against a platinum auxiliary electrode and a silver/silver chloride reference electrode by applying a $0 \mathrm{~V}$ potential for 2 seconds followed by $0.3 \mathrm{~V}$ for 2 seconds, for 5 cycles. This procedure was repeated for all electrode types and relevant glucose concentrations. After sensing, the electrodes were cleaned with $5 \mathrm{CV}$ cycles between 0.2 and $1.8 \mathrm{~V}$ in a $500 \mathrm{mM}$ sulfuric acid solution.

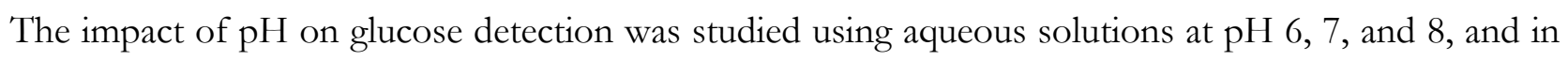
$0.1 \mathrm{M}$ phosphate buffer $\mathrm{pH}$ 7.4. For these experiments, a $20 \mathrm{mM}$ glucose solution was prepared in the previously described buffer and a cyclic voltammogram (between -0.1 and $0.7 \mathrm{~V}$ at $0.1 \mathrm{~V} / \mathrm{s}$ ) and chronoamperogram were obtained.

\section{Results and Discussion}

Fabrication of nanoroughened electrodes

A prevailing challenge associated with the miniaturization of electrochemical sensors is the reduction of sensitivity when conventional planar electrodes are used. This is due to the reduced physical and electrochemical active surface area when the electrode is reduced in size. To achieve miniaturization while improving sensitivity in portable sensing chips, we have used a shape-memory polymer thin film structuring approach $[21,22]$ and an electrochemical roughening technique to significantly enhance the electroactive surface area of microstructured electrodes (MSEs). Figure 1 depicts the fabrication process and the subsequent modification through electrochemical pulsing that results in roughened MSEs (R-MSEs).

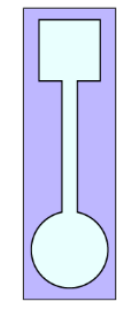

Masked PS
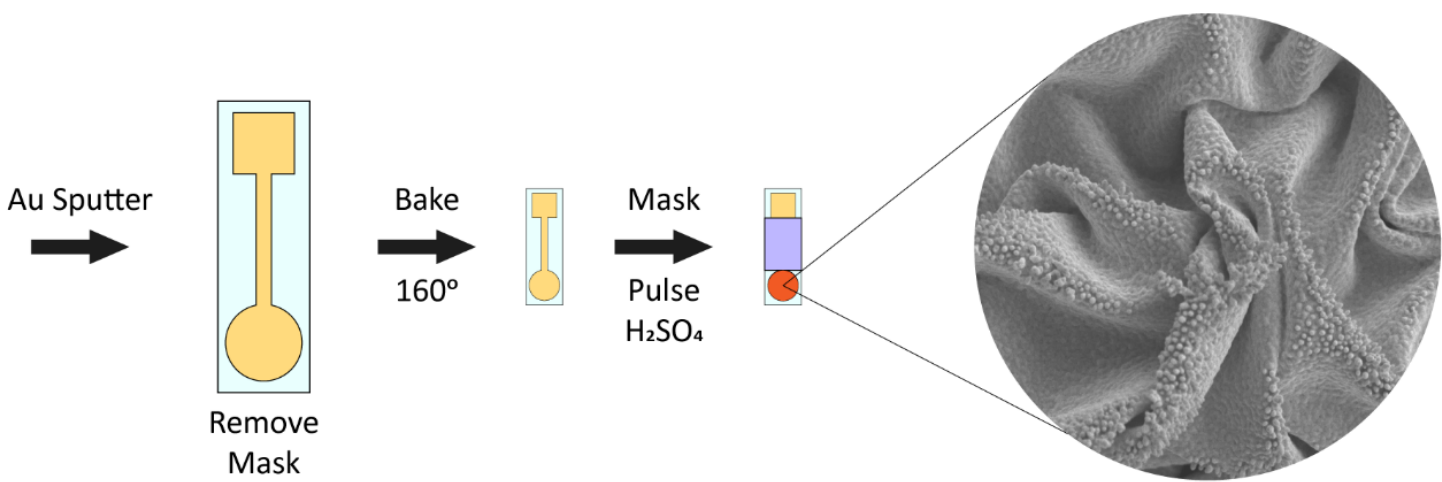

Figure 1. Illustration of the MSE fabrication and electrochemical roughening processes. Inset shows an electron micrograph of an MSE in the early stages of roughening. 
The electrodes were fabricated ( $c f$. Materials and Methods) on pre-stressed polystyrene sheets that were cleaned in a series of solvent washes (ethanol, isopropanol and water, in sequence), masked to define the electrode shapes and cut into individual electrodes. Gold was sputter-deposited onto the substrate, after which the mask was removed, yielding patterned gold electrodes. The substrates containing the electrodes were then heated in an oven at $160{ }^{\circ} \mathrm{C}$ to shrink down to $\sim 16 \%$ of their original geometric area. The result were MSEs with electroactive surface area (ESA) 6x greater than their planar counterpart, as previously reported.[21, 22] From this point onward, limited cost-effective options are available for further enhancement of the ESA. Among these are the approaches of metallic salt or nanoparticle electrodeposition, and alloy deposition followed by a chemical etching process.[24-26] . However, such options require additional materials during the electrode fabrication process or generate waste, making them impractical for use in resource-limited settings. To enhance the ESA post-shrinking while circumventing material waste and increased production cost, we have used a electrochemical roughening approach similar to that reported by the group of Plaxco.[27]. In this process, the MSE is placed in a concentrated sulfuric acid solution and a potential step is applied between 0 and $2 \mathrm{~V}$, with a $20 \mathrm{~ms}$ dwell time at each potential. At a potential of $2 \mathrm{~V}$, gold atoms of the MSE surface are locally dissolved and upon potential stepping to $0 \mathrm{~V}$ the gold atoms are redeposited back onto the electrode. This process creates nanometeric pores and protrusions on the gold electrode surface, which can be visualized through scanning electron microscopy (SEM, Figure 1). Further gold dissolution and re-deposition with subsequent pulsing cycles results in an evolution from small bumps, produced during the initial pulsing phase, into larger surface features; this effectively increases the degree of roughening and consequently the ESA relative to pristine MSEs.

\section{Assessment of electroactive surface area}

The ESA of nanoroughened MSEs was evaluated through CV by the oxidation of the gold surface in an acidic solution. Two concentrations of sulfuric acid were first compared to determine the best conditions to assay the ESA. We found that the reduction current peak in $50 \mathrm{mM} \mathrm{H}_{2} \mathrm{SO}_{4}$ was lower and broader than those obtained in $500 \mathrm{mM} \mathrm{H}_{2} \mathrm{SO}_{4}$ (Figure S1A). However, the integration of the cathodic peak current showed that conducting the assay in either 50 or $500 \mathrm{mM} \mathrm{H}_{2} \mathrm{SO}_{4}$ resulted in similar charge transfer, which confirms that the ESA remains unchanged upon change of the concentration of the acidic solution (Figure S1B). We next compared the efficiency of the pulsing procedure when performed under different $\mathrm{H}_{2} \mathrm{SO}_{4}$ concentrations. Pulsing in $50 \mathrm{mM} \mathrm{H}_{2} \mathrm{SO}_{4}$ resulted in electrodes with lower ESAs compared to those pulsed in $500 \mathrm{mM} \mathrm{H}_{2} \mathrm{SO}_{4}$ (Figure S1C). Based on these findings, all 
subsequent experimentation was carried out using electrodes where both the nano-roughening process and the evaluation of the ESA were carried out in $500 \mathrm{mM} \mathrm{H}_{2} \mathrm{SO}_{4}$ solutions.

Another important aspect in the fabrication of nanostructured electrodes is the ability to maintain their structural integrity during drying and storage. We evaluated the impact of the drying step after roughening in solution by comparing R-MSEs that were subjected to oven-, air- and nitrogen-drying. Figure S2A-C shows cyclic voltammograms for roughened electrodes that have gone through each drying approach, overlaid with CV data for non-roughened MSEs and roughened electrodes prior to the drying step. From these results it is evident that air- and nitrogen-drying were better at maintaining the roughened 3D features on the surface intact than oven drying, as their signal output was comparable to that of R-MSEs before drying. Conversely, oven-dried samples showed reduced signal that was similar to that of MSEs pre-roughening, which suggests that the 3D nanostructures produced during roughening were destroyed during the oven-drying process. The SEM images in Figure S2D show the topographical features of each R-MSE after being subjected to different amounts of pulsing and drying conditions. These images confirm that the structures for oven dried materials have lower roughness and less prominent topography, leading to lower measured ESA values. Considering that the air-drying method is more time consuming and depends strongly on relative humidity conditions, nitrogen flow was selected as the preferred drying method for R-MSEs.

To validate that the 3D nanostructures formed via electrochemical roughening enhanced the performance of MSEs, CV was performed in sulfuric acid, and we compared the ESA of planar gold electrodes, MSEs and R-MSEs (Figure 2). The geometrical footprint was kept constant by applying a vinyl mask with a circular cut-out over the working pad of each electrode. Figure 2A shows representative cyclic voltammograms obtained, where the signal generated from the forward scan (positive direction) represents the anodic current and the reverse scan (negative direction) represents the cathodic current. As expected, the cathodic peak for each electrode type appears at the same potential, corresponding to the reduction of the gold oxide surface, with R-MSEs exhibiting a much larger current than MSEs and planar electrodes. The total charge transferred per CV cycle for each type of electrode was calculated by integrating the cathodic peak. This value was subsequently divided by a factor of $386 \mu \mathrm{C} / \mathrm{cm}^{2}$ (estimated charge required to oxidize the gold surface), which yielded the ESA.[28] The ESA of each type of electrode was measured for three independently fabricated electrodes to provide statistical variance. 

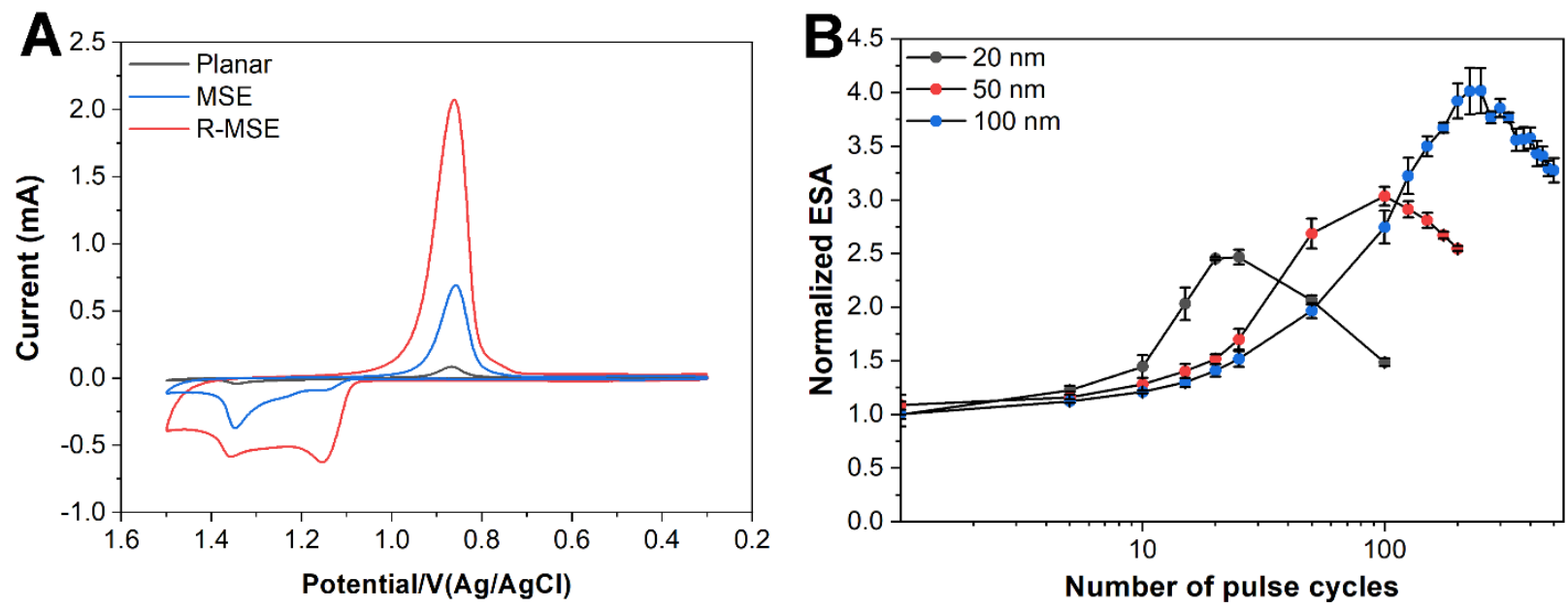

Figure 2. A) CV overlay showing differences between planar, MSE, and R-MSE fabricated with 200 pulse cycles. B) ESA as a function of the number of pulse cycles used to roughen MSEs of different thickness. ESA was normalized to MSEs with 0 pulses applied. Error bars represent the standard deviation obtained from three independently fabricated electrodes.

Figure 2B shows normalized ESA measurements for 20, 50, and $100 \mathrm{~nm}$ thick MSEs at increasing number of electrochemical roughening pulse cycles. For all gold thicknesses tested, a trend can be observed in which the ESA of the MSE increases with the number of pulse cycles, up to a maximum that is characteristic to the thickness of the gold film $(25,100$, and 200 pulses for 20, 50 and $100 \mathrm{~nm}$ thick electrode, respectively). Additional pulse cycles after this point lead to a reduction in ESA, with thicker gold allowing a larger ESA maxima prior to decay. We hypothesized that the reduction in ESA was a result of the loss of structural stability and eventual collapse of the gold nanostructures formed during the electrochemical roughening process. This was confirmed by SEM images obtained from electrodes of all thicknesses and roughening cycles ranging from $0-200$ pulses, as described below.

\section{Nanostructure characterization}

Gold electrodes that are made through sputter coating and subjected to thermal shrinking contained nanometer- to micrometer-sized wrinkles, whose size is dependent on the thickness of the deposited gold film. The surface of these wrinkles is always smooth, regardless of the thickness of the thin film. However, when electrochemical pulses are applied, 3D structures of nanometric size grow on the MSE surface (Figure 3). The roughening process is inhomogeneous, with most nanostructures growing on the tips of the wrinkles, independently of gold thickness. This is attributed to the electric field enhancement at the sharply curved surfaces presented by the wrinkle peaks.[21] Increasing the number 
of pulses results in larger 3D nanofeatures in the electrodes up to a threshold after which the surface appears less rough. SEM images of the R-MSEs explain the behavior observed from the ESA assessment (Figure 2B), where after a threshold number of pulses - characteristic to each gold film thickness - the structures that comprise the surface roughness will collapse, reducing the ESA. The decrease in ESA is exacerbated by progressive loss of material to solution and the eventual exposure of the non-conductive PS surface. For example, a $20 \mathrm{~nm}$ Au electrode subjected to 50 pulses was riddled with pinholes; additional pulses resulted in further damage to the gold surface, at which point the ESA was lower than that observed after applying 10 pulses (Figure 2B). For an electrode of $50 \mathrm{~nm} \mathrm{Au}$ thickness, electrode damage and decreasing ESA was observed after 100 pulses, while $100 \mathrm{~nm} \mathrm{Au}$ electrodes were more resilient, retaining stable 3D structures devoid of pinholes up to 200 pulses. These observations conform well with the trends in ESA extracted through CV. Considering these results, the R-MSE electrodes evaluated for the applications discussed below were fabricated from 100 nm-thick MSEs roughened with 200 pulses.

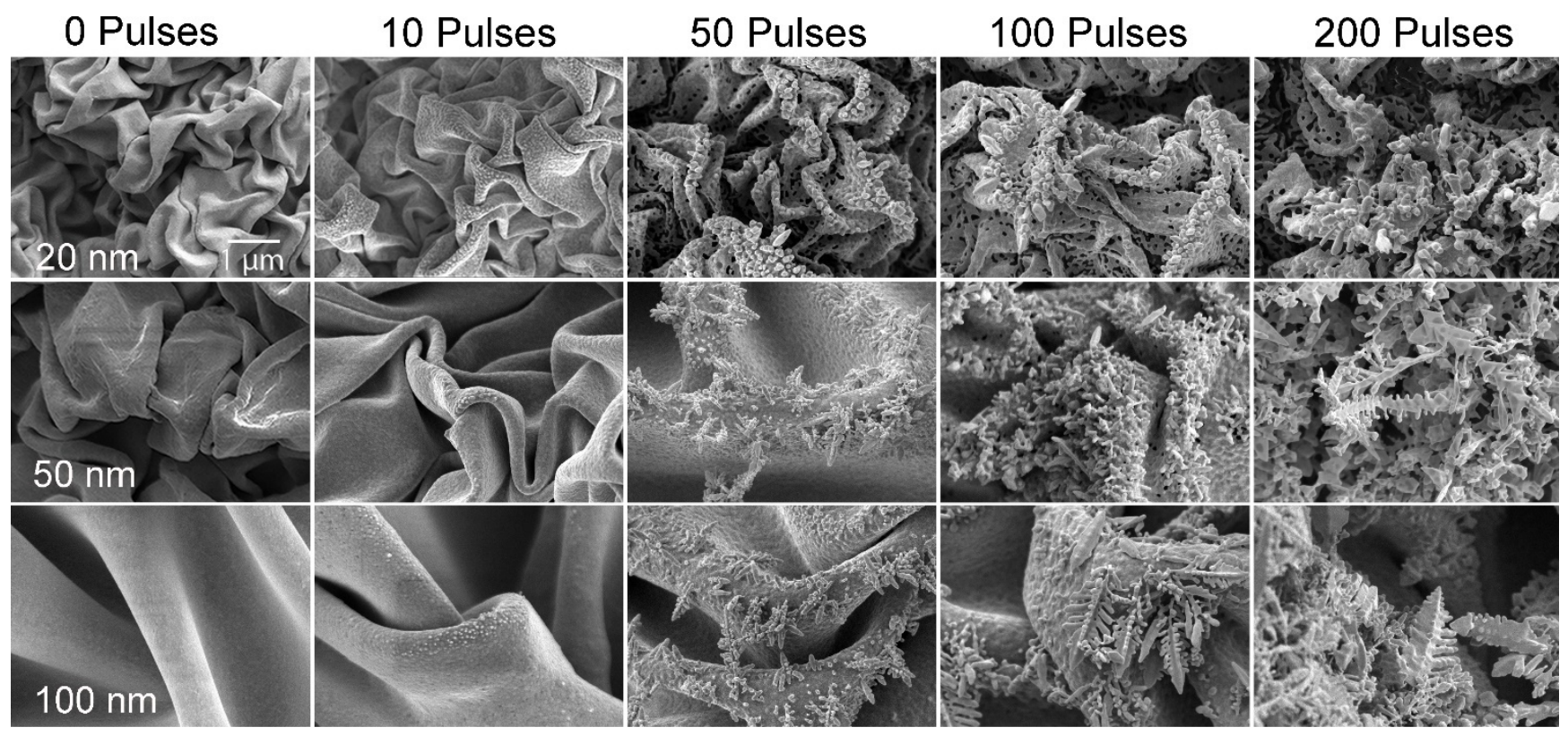

Figure 3. Representative SEM images of electrodes subjected to increasing number of roughening pulse cycles.

\section{Anti-fouling properties of nanoroughened electrodes}

Biofouling poses a considerable challenge on of biosensor development because it prevents the analyte of interest from reaching the active surface of a sensing device. Biofouling can be driven by a number of mechanisms including the hydrophobicity of the surface, which plays a major role in the adsorption of biofouling agents. It has been reported that increasing the hydrophobicity of a substrate can enhance 
the surface adsorption of some proteins. [29] In the case of human serum albumin (HSA), contact with an adsorbent surface prompts the denaturation of the protein, and can lead to the formation of a multilayer of unfolded peptides that obstructs contact between a molecule of interest and the active surface of an electrode.[18, 29, 30] Several works have reported a gradual reduction of the electrochemical signal generated from gold electrodes when an analyte is detected in a biofluid. This decline of signal is mostly attributed to the presence of biofouling proteins.[17, 18, 31] HSA is the main protein constituent of human blood, with typical concentrations ranging between 35 and $54 \mathrm{mg} / \mathrm{mL}$, and the main biofouling agent when detecting species in blood. A protein that is frequently used to model biofouling in biological media is bovine serum albumin (BSA), a homologue of HSA. To evaluate the antifouling properties of the fabricated electrodes, we measured the signal obtained from sensing a redox active compound (KFeCy) through CV using planar, MSE and R-MSE gold electrodes in presence of BSA at different concentrations. By exposing the electrode surface to BSA, we can simulate the degree of biofouling that is expected in clinically relevant samples such as urine, blood and serum. Microstructuring and nano-roughening processes significantly increase the contact angle of water droplets on the structured electrodes (Figure 4A). The behavior of these electrode surfaces conforms to the Cassie-Baxter model of surface wettability, where the contact angle of a liquid droplet increases with the fraction of air $(\sigma)$ entrapped by the surface topography.[32] The fraction of air can be calculated as $\sigma=\left(\cos \theta_{r}-\cos \theta\right) /(\cos \theta+1)$, where $\theta_{r}$ is the contact angle of the modified surface and $\theta$ is the contact angle of the unstructured surface. MSEs and R-MSEs presented values for $\sigma$ of 0.82 and 0.90 , respectively. While this would suggest that the adsorption of biofouling proteins should be higher for the R-MSE electrodes, we found that this type of electrode surface possesses a excellent anti-fouling capacity against BSA.

We evaluated the antifouling capabilities of flat electrodes, MSEs and R-MSEs by quantifying the decrease in CV signal obtained from a solution containing $10 \mathrm{mM} \mathrm{KFeCy}$ and $2 \mathrm{mg} / \mathrm{mL}$ BSA over time intervals of up to 30 minutes (Figure S3). The behavior of the three electrode surface types is shown in Figure 4B. In line with expectations for planar electrodes, their immersion in the solution led to near-complete suppression of the signal recorded from $\mathrm{KFeCy}$, arising from to the formation of an anionic BSA layer that repels $\mathrm{KFeCy}$ - a negatively charged analyte. MSEs exhibited a partial loss of signal, with $\sim 35 \%$ of the faradaic current remaining after 5 minutes in the solution. The resistance to biofouling could reflect the MSEs having a 3-dimensional topography and a surface area that is 6x larger than that of the planar electrodes, therefore requiring more time for a layer of BSA to coat the surface 
in its entirety. This is supported by the fact that after 20 minutes in the solution, there was no signal recorded from MSEs (Figure S3B). Conversely, R-MSEs retained signal producing capability exceptionally well, with $\sim 95 \%$ remaining after 30 minutes of immersion in the $2 \mathrm{mg} / \mathrm{mL}$ BSA solution (Figure S3C). Even at a greater BSA concentration of $40 \mathrm{mg} / \mathrm{mL}$ (comparable to that of HSA in blood), $>50 \%$ of signal was recorded after the electrodes were immersed in the solution for 10 minutes (Figure 4C). This resistance to fouling cannot be accounted by differences in surface area alone - a different explanation is needed.
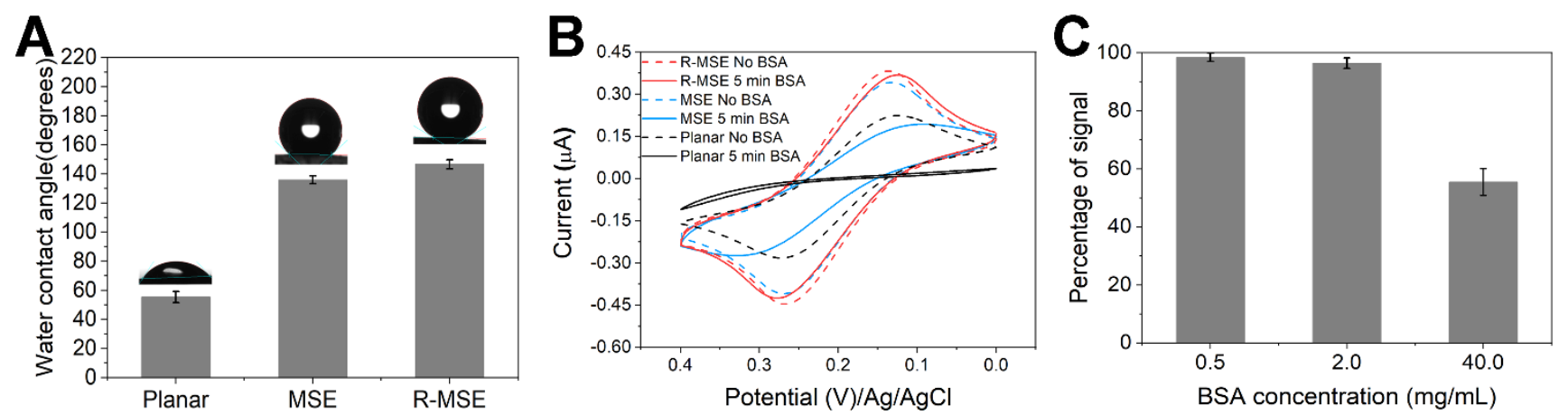

Figure 4. A) Water contact angle on planar electrodes, MSEs and R-MSEs. B) Cyclic voltammograms obtained from planar electrodes, MSE, and R-MSEs incubated in a $2.0 \mathrm{mg} / \mathrm{mL}$ BSA solution for $10 \mathrm{~min}$ and used to sense a solution containing $10 \mathrm{mM} \mathrm{KFeCy.} \mathrm{Dashed} \mathrm{lines} \mathrm{represent} \mathrm{the} \mathrm{respective} \mathrm{controls} \mathrm{run} \mathrm{without}$ BSA incubation. C) Relative peak area of the CVs obtained from R-MSEs incubated for $10 \mathrm{~min} \mathrm{in} 0.5 \mathrm{mg} / \mathrm{mL}$, $2.0 \mathrm{mg} / \mathrm{mL}$ and $40 \mathrm{mg} / \mathrm{mL}$ BSA solutions, normalized to the signal obtained from bare R-MSEs.

In 2013, Collinson et al.[17] tested for the first time the antifouling capabilities of nanoporous gold (NPG) electrodes. They observed - using KFeCy as a redox probe - high antifouling capabilities of NPGs when challenged with BSA at a concentration of $2 \mathrm{mg} / \mathrm{mL}$. This behaviour was attributed to two contributing factors, other than the increase in surface area. First, they hypothesized that given the different sizes of the protein $(4 \times 14 \mathrm{~nm})$ and the redox probe $(<1 \mathrm{~nm})$, mass transport limitations are relevant. The size difference hinders access of BSA to the inner surfaces of the porous gold surface, while KFeCy faces no such limitations. Second, they asserted that the roughness, porosity, and nanoscale architecture (curvature) of the NPG electrodes render the blocking of the surface by protein adsorption and/or unfolding less effective. These considerations can be applied to our electrode surfaces, where the nanoscale features on the R-MSE surface are in such close proximity that they can prevent the penetration of BSA into the small pores created in the gold film but allow the smaller redox reporter molecules to enter. Similarly, the nanoscale surface roughness can prevent or hinder the 
adsorption and unfolding of BSA. A third explanation for antifouling behaviour in nanostructured electrodes, presented in the work of Seal et al.,[18] relates to the electrical double layer (EDL) nanoexclusion phenomenon. This work suggests that the EDL within nanopores imparts an additional force that repels anionic BSA molecules from adsorbing to the surface. A similar effect is expected in R-MSEs, as the EDL associated with the 3D nanostructures on the gold surface could repel BSA but allow the access of KFeCy to the sensor's surface. Regardless on its origins, the anti-fouling behaviour of R-MSEs makes them promising for the development of electrochemical sensors for the detection of analytes in biofluids.

\section{Heavy metal sensing using R-MSEs}

A prime application for electrochemical sensing technology is the detection of heavy metals. The high ESA and remarkable antifouling properties of R-MSE electrodes makes them excellent candidates to detect heavy metals in real water samples. Current approaches rely on adsorption spectroscopy techniques, which are time-consuming, expensive and often require complex pretreatment of samples.[33] This is in stark contrast to electrochemical detection approaches which constitute inexpensive and simpler alternative routes. [34] The most common among these methods is the deposition of ions of the target metal at an underpotential in combination with anodic stripping voltammetry (UPD-ASV).[35] We evaluated the use of planar, microstructured and roughenedmicrostructured gold electrodes for copper detection by UPD-ASV. In these experiments, $\mathrm{Cu}^{2+}$ was deposited at an underpotential of $-0.1 \mathrm{~V}$ for 180 seconds to allow the formation of a monolayer of solid copper. ASV was subsequently performed by means of linear sweep voltammetry. The results are presented in Figures 5 and $\mathbf{S} 4$. 


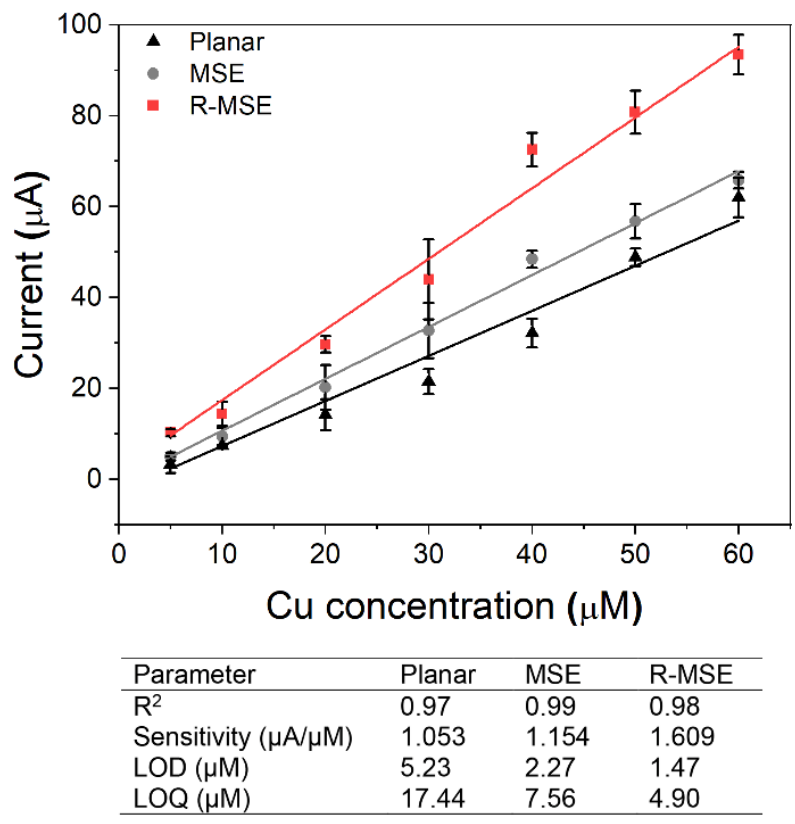

Figure 5. Copper sensing through UPD-ASV using planar Au electrodes, MSEs and R-MSEs.

R-MSEs presented a significantly lower limit of detection (LOD), lower limit of quantification (LOQ) and higher sensitivity than planar electrodes and MSEs. These differences likely arise from the enhanced ESA of R-MSEs, which provides a greater accessible surface for the electrodeposition of copper. Indeed, a higher current output is obtained from R-MSEs than from planar electrodes and MSEs under the same experimental conditions. The LOQ for the R-MSE was $5 \mu \mathrm{M}$, equivalent to $0.3 \mathrm{ppm}$. The maximum permitted content of copper in tap water is 2 and $1.3 \mathrm{ppm}$ in Canada and the U.S., respectively; the LOQ of the nanoroughened electrodes qualifies them with distinction for possible use in the electrochemical sensing of copper in tap water. Considering the high antifouling ability of the R-MSE, these electrodes demonstrate potential for the detection of copper even in polluted water replete with fouling agents. To identify the full extent of the heavy metal sensing capabilities of these modified electrode surfaces, further assessment of their detection of different metals and the implementation of more sensitive electrochemical techniques - such as square wave voltammetry or differential pulse voltammetry - may prove useful.

\section{Enzyme-free glucose sensing}

Undoubtedly, the most successful application of electrochemical biosensing is embodied by the now widely available glucose meters. Current commercially available devices rely on the enzymatic oxidation of glucose to generate hydrogen peroxide, a species that can be detected on the surface of the sensing 
electrode or indirectly by the consumption of the oxygen in the solution. Despite their effectiveness and widespread use, this technology is hindered by the nature of enzymes - biological entities that are sensitive to and may denature upon exposure to environmental conditions like increased temperature. For this reason, the use of enzyme-free working electrodes in glucose sensing has emerged as a very active field in electrochemistry, where structured gold electrodes seem to be an attractive option.[15, 16, 36]

The ability of planar electrodes, MSEs, and R-MSEs to oxidize glucose was studied through chronoamperometry at three different $\mathrm{pH}$ values $(6,7$ and 8$)$ in phosphate buffer. We observed that for all types of electrodes the current obtained from these measurements increased as the $\mathrm{pH}$ increased (Figure S5). Previous work has shown that the formation of adsorbed gold hydroxide $\left(\mathrm{Au}(\mathrm{OH})_{\text {ads }}\right)$ plays a major role in the electro-oxidation mechanism of glucose on gold surfaces.[36, 37] Therefore we propose that an increase in $\mathrm{pH}$ promotes $\mathrm{Au}(\mathrm{OH})_{\text {ads }}$ formation and enhances the electro-oxidation of glucose, giving rise to the observed increase in current. At all $\mathrm{pH}$ levels, we observed that the current obtained for R-MSEs was higher than that of their MSE and planar electrode counterparts.

Chloride has been identified as the most prominent source of interference in enzyme-free glucose detection using gold sensors. [15, 38] Chloride ions readily adsorb onto the gold surface, blocking active sites that would otherwise be involved in the formation of $\mathrm{Au}(\mathrm{OH})_{\text {ads. }}$ The electro-oxidation of glucose in 1x PBS - which contains chloride at a concentration similar to that in human blood - was evaluated for the three electrode types. As a reference, chloride-free phosphate buffer at the same $\mathrm{pH}$ was also used in electrochemical analysis. Unsurprisingly, lower output current was obtained from electrodes in 1x PBS than in chloride-free phosphate buffer (Figure S6); yet, the reduced current remained sufficient for the generation of a calibration curve for the electro-oxidation of glucose (Figure 6). The LOD and LOQ obtained from R-MSEs were 0.53 and $1.78 \mathrm{mM}$, respectively - approximately 3-fold lower than those for the MSEs, while planar electrodes did not show any catalytic activity. Given that typical glucose blood levels for healthy adults range from 3-7 mM, the LOQ obtained for R-MSEs combined with the high antifouling capacity of these electrodes set these sensors forth as a promising alternative for inexpensive enzyme-free glucose sensing. 


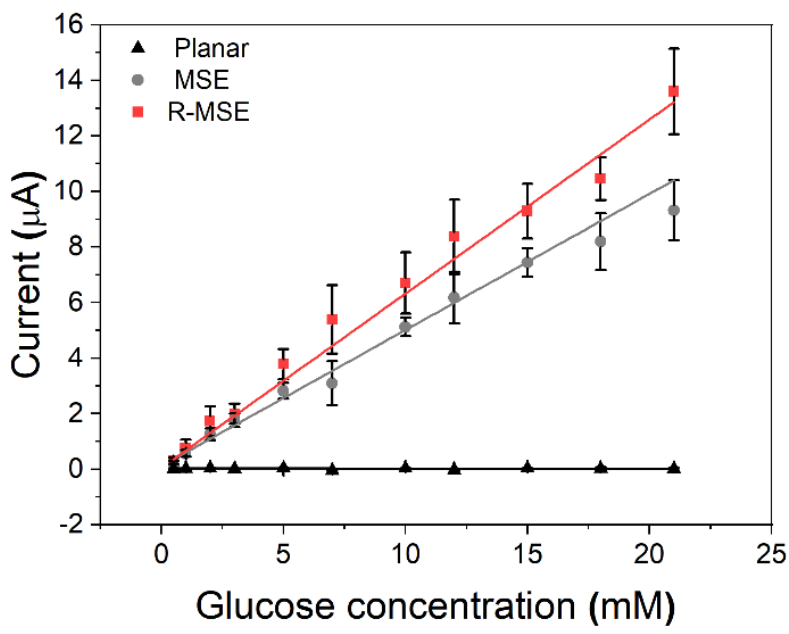

\begin{tabular}{lcll}
\hline Parameter & Planar & MSE & R-MSE \\
\hline$R^{2}$ & - & 0.99 & 0.98 \\
Sensitivity $(\mu \mathrm{A} / \mathrm{mM})$ & - & 0.4517 & 0.6107 \\
LOD $(\mathrm{mM})$ & - & 1.37 & 0.53 \\
LOQ $(\mathrm{mM})$ & - & 4.57 & 1.78 \\
\hline
\end{tabular}

Figure 6. Measurement of glucose concentration through direct glucose oxidation using planar Au electrodes, MSEs and R-MSEs in 1x PBS buffer.

In the context of in vivo glucose detection, ascorbic acid (AA) accounts for a large proportion of signal interference. To evaluate susceptibility to this interferant, we compared the responses of MSEs and RMSEs to $\mathrm{AA}$ at a concentration of $20 \mathrm{mM}$ in $1 \mathrm{x}$ PBS - notably higher than the expected AA concentration in blood, which is in the $20-60 \mu \mathrm{M}$ range. Figure S7, shows that the difference in anodic peak current $\left(\mathrm{i}_{\mathrm{pa}}\right)$ between R-MSEs and MSEs is less pronounced for the electro-oxidation of AA than for glucose under the same experimental conditions. In fact, the quotient of $i_{\text {pa-R-MSE }} / i_{\text {pa-MSE }}$ for AA electro-oxidation was calculated to be 1.1, whereas for glucose it yielded a quotient of 2.3. This implies that the difference in nanotopography and ESA between these electrode surfaces has a greater impact on the ability to detect glucose than on AA sensing. This can be explained based on the processes that control electron transfer between the analyte and the electrode surface. AA relies on a diffusioncontrolled process, meaning that the increase in ESA has no noticeable impact on the detection of this analyte: the velocity of the associated electronic transfer is too fast, and much of the electroactive surface is not readily accessed by the AA molecules. In contrast, the slowest step in glucose detection is the kinetically controlled electron transfer between the molecule and the gold surface. In consequence, the majority of the ESA is accessible to the analyte, leading to a substantial increase in the output current of R-MSEs relative to MSEs. A second factor enhances the ability to detect glucose with R-MSEs is the dominant crystalline facet presented by the gold surface. It is known that the highest current generated 
in the electro-oxidation process of glucose is obtained from surfaces rich in (111) and (100) facets.[39] Jeyabharathi et al.[40] reported that the oxidation peaks obtained through CV performed on gold electrodes immersed in sulfuric acid can be indexed to the different facets, with three main peaks at 1.14, 1.2, and 1.35 V corresponding to facets (100), (110) and (111), respectively. CV obtained in sulfuric acid (Figure 2A) for planar electrodes, MSEs and R-MSEs show that R-MSEs are rich in facets (110) and (111), which contribute to their superior performance in glucose detection. Considering the high current output produced by the R-MSE in the electrooxidation of glucose and their specificity toward this molecule they could be a breakthrough in the fabrication of cost-effective and highly sensitive electrochemical sensors for the enzyme-free detection of glucose.

\section{Conclusion}

In this work, we have introduced a simple methodology to fabricate high surface area roughened microstructured electrodes (R-MSEs), where through chronoamperometric pulsing microstructured gold electrodes are roughened by the local dissolution and redeposition of gold ions into 3D nanostructures. The electroactive surface area (ESA) of R-MSEs is increased by up to 5-fold, compared with microstructured electrodes, and approximately 30-fold relative to planar electrodes. We found a direct correlation between the thickness of the thin gold film and the maximum number of pulsing cycles that a given electrode can withstand prior to the collapse of the nanostrucutres and a decay of ESA. The R-MSEs obtained from the current-pulsing process exhibit excellent antifouling capacity, making these newly fabricated electrodes an attractive option for biosensing in complex biofluids. The ability of R-MSEs to detect copper was explored, yielding an LOQ of $0.3 \mathrm{ppm}$. Furthermore, highly sensitive enzyme-free detection of glucose was demonstrated in 1x PBS with an LOQ of $1.78 \mathrm{mM}$, which provides sensing capabilities compatible with typical glucose levels in human blood. Considering in tandem the LOQ for glucose sensing and the high antifouling capacity of R-MSEs, these electrodes offer potential for the development of an inexpensive and easily fabricated enzyme-free glucose sensing system. The R-MSE electrodes also open the door for other types of sensing systems that leverage the functionalization of the nanostructures to provide highly sensitive detection of DNA and small molecule binding events.

\section{Acknowledgements}

Eduardo Gonzalez Martinez was partially supported by an International Ontario Graduate Scholarship and Nadine E. Beganovic was partially supported through a Natural Sciences and Engineering Research Council (NSERC) of Canada Undergraduate Student Research Award. Jose Moran-Mirabal is the Tier 
2 Canada Research Chair in Micro and Nanostructured Materials and the recipient of an Early Researcher Award from the Ontario Ministry of Research and Innovation. The research presented was supported by funding through a Discovery Grant from NSERC to JMM (RGPIN-2019-06433). This research made use of instrumentation available through the Canadian Centre for Electron Microscopy at McMaster University.

\section{References}

[1] M. Majdinasab, K. Mitsubayashi, J.L. Marty, Optical and Electrochemical Sensors and Biosensors for the Detection of Quinolones, Trends in Biotechnology 37(8) (2019) 898-915.

[2] T. Chaibun, C. La-o-vorakiat, A.P. O’Mullane, B. Lertanantawong, W. Surareungchai, Fingerprinting Green Curry: An Electrochemical Approach to Food Quality Control, ACS Sensors 3(6) (2018) 1149-1155.

[3] M. Bariya, Z. Shahpar, H. Park, J. Sun, Y. Jung, W. Gao, H.Y.Y. Nyein, T.S. Liaw, L.-C. Tai, Q.P. Ngo, M. Chao, Y. Zhao, M. Hettick, G. Cho, A. Javey, Roll-to-Roll Gravure Printed Electrochemical Sensors for Wearable and Medical Devices, ACS Nano 12(7) (2018) 6978-6987.

[4] A.R. Jalalvand, M.M. Zangeneh, F. Jalili, S. Soleimani, J.M. Díaz-Cruz, An elegant technology for ultrasensitive impedimetric and voltammetric determination of cholestanol based on a novel molecularly imprinted electrochemical sensor, Chemistry and Physics of Lipids 229 (2020) 104895.

[5] X. Wang, Y. Qi, Y. Shen, Y. Yuan, L. Zhang, C. Zhang, Y. Sun, A ratiometric electrochemical sensor for simultaneous detection of multiple heavy metal ions based on ferrocene-functionalized metal-organic framework, Sensors and Actuators B: Chemical 310 (2020) 127756.

[6] Z. Liu, H. Ma, H. Sun, R. Gao, H. Liu, X. Wang, P. Xu, L. Xun, Nanoporous gold-based microbial biosensor for direct determination of sulfide, Biosensors and Bioelectronics 98 (2017) 29-35.

[7] Y. Zhao, X. Zheng, Q. Wang, T. Zhe, Y. Bai, T. Bu, M. Zhang, L. Wang, Electrochemical behavior of reduced graphene oxide/cyclodextrins sensors for ultrasensitive detection of imidacloprid in brown rice, Food Chemistry 333 (2020) 127495.

[8] F. Schröper, D. Brüggemann, Y. Mourzina, B. Wolfrum, A. Offenhäusser, D. Mayer, Analyzing the electroactive surface of gold nanopillars by electrochemical methods for electrode miniaturization, Electrochimica Acta 53(21) (2008) 6265-6272.

[9] K. Murata, K. Kajiya, M. Nukaga, Y. Suga, T. Watanabe, N. Nakamura, H. Ohno, A Simple Fabrication Method for Three-Dimensional Gold Nanoparticle Electrodes and Their Application to the Study of the Direct Electrochemistry of Cytochrome c, Electroanalysis 22(2) (2010) 185-190.

[10] P.N. Bartlett, J.J. Baumberg, P.R. Birkin, M.A. Ghanem, M.C. Netti, Highly Ordered Macroporous Gold and Platinum Films Formed by Electrochemical Deposition through Templates Assembled from Submicron Diameter Monodisperse Polystyrene Spheres, Chemistry of Materials 14(5) (2002) 2199-2208.

[11] H. Lu, Y. Liu, G.-C. Han, Optical and electrochemical properties of ordered macroporous gold array on the ITO surface, Applied Surface Science 257(16) (2011) 7030-7035.

[12] S. Marx, M.V. Jose, J.D. Andersen, A.J. Russell, Electrospun gold nanofiber electrodes for biosensors, Biosensors and Bioelectronics 26(6) (2011) 2981-2986.

[13] J. van der Zalm, S. Chen, W. Huang, A. Chen, Review-Recent Advances in the Development of Nanoporous Au for Sensing Applications, Journal of The Electrochemical Society 167(3) (2020) 037532.

[14] T. Fujita, P. Guan, K. McKenna, X. Lang, A. Hirata, L. Zhang, T. Tokunaga, S. Arai, Y. Yamamoto, N. Tanaka, Y. Ishikawa, N. Asao, Y. Yamamoto, J. Erlebacher, M. Chen, Atomic origins of the high catalytic activity of nanoporous gold, Nature materials 11(9) (2012) 775-80. 
[15] L.Y. Chen, X.Y. Lang, T. Fujita, M.W. Chen, Nanoporous gold for enzyme-free electrochemical glucose sensors, Scripta Materialia 65(1) (2011) 17-20.

[16] Y. Pei, M. Hu, F. Tu, X. Tang, W. Huang, S. Chen, Z. Li, Y. Xia, Ultra-rapid fabrication of highly surfaceroughened nanoporous gold film from AuSn alloy with improved performance for nonenzymatic glucose sensing, Biosensors and Bioelectronics 117 (2018) 758-765.

[17] J. Patel, L. Radhakrishnan, B. Zhao, B. Uppalapati, R.C. Daniels, K.R. Ward, M.M. Collinson, Electrochemical Properties of Nanostructured Porous Gold Electrodes in Biofouling Solutions, Analytical Chemistry 85(23) (2013) 11610-11618.

[18] S. Saraf, C.J. Neal, S. Park, S. Das, S. Barkam, H.J. Cho, S. Seal, Electrochemical study of nanoporous gold revealing anti-biofouling properties, RSC Advances 5(58) (2015) 46501-46508.

[19] T.A. Silva, M.R.K. Khan, O. Fatibello-Filho, M.M. Collinson, Simultaneous electrochemical sensing of ascorbic acid and uric acid under biofouling conditions using nanoporous gold electrodes, Journal of Electroanalytical Chemistry 846 (2019) 113160.

[20] P.-H. Lin, B.-R. Li, Antifouling strategies in advanced electrochemical sensors and biosensors, Analyst 145(4) (2020) 1110-1120.

[21] C.M. Gabardo, Y. Zhu, L. Soleymani, J.M. Moran-Mirabal, Bench-Top Fabrication of Hierarchically Structured High-Surface-Area Electrodes, Advanced Functional Materials 23(24) (2013) 3030-3039.

[22] M. Abbaszadeh Amirdehi, S. Saem, M.P. Zarabadi, J.M. Moran-Mirabal, J. Greener, Microstructured Anodes by Surface Wrinkling for Studies of Direct Electron Transfer Biofilms in Microbial Fuel Cells, Advanced Materials Interfaces 5(13) (2018) 1800290.

[23] S. Saem, O. Shahid, A. Khondker, C. Moran-Hidalgo, M.C. Rheinstädter, J. Moran-Mirabal, Benchtopfabricated lipid-based electrochemical sensing platform for the detection of membrane disrupting agents, Scientific Reports 10(1) (2020) 4595.

[24] X. Xiao, P. Si, E. Magner, An overview of dealloyed nanoporous gold in bioelectrochemistry, Bioelectrochemistry 109 (2016) 117-126.

[25] N. Wongkaew, M. Simsek, C. Griesche, A.J. Baeumner, Functional Nanomaterials and Nanostructures Enhancing Electrochemical Biosensors and Lab-on-a-Chip Performances: Recent Progress, Applications, and Future Perspective, Chemical Reviews 119(1) (2019) 120-194.

[26] M. Collinson, Nanoporous gold electrodes and their applications in analytical chemistry, ISRN Anal. Chem. 2013 (2013).

[27] N. Arroyo-Currás, K. Scida, K.L. Ploense, T.E. Kippin, K.W. Plaxco, High Surface Area Electrodes Generated via Electrochemical Roughening Improve the Signaling of Electrochemical Aptamer-Based Biosensors, Analytical Chemistry 89(22) (2017) 12185-12191.

[28] S. Trasatti, O.A. Petrii, Real surface area measurements in electrochemistry, Pure and Applied Chemistry 63(5) (1991) 711-734.

[29] A. Barfidokht, J.J. Gooding, Approaches Toward Allowing Electroanalytical Devices to be Used in Biological Fluids, Electroanalysis 26(6) (2014) 1182-1196.

[30] K. Nakanishi, T. Sakiyama, K. Imamura, On the adsorption of proteins on solid surfaces, a common but very complicated phenomenon, Journal of Bioscience and Bioengineering 91(3) (2001) 233-244.

[31] P. Daggumati, Z. Matharu, L. Wang, E. Seker, Biofouling-Resilient Nanoporous Gold Electrodes for DNA Sensing, Analytical Chemistry 87(17) (2015) 8618-8622.

[32] L. Jing, Y.-e. Shi, J. Cui, X. Zhang, J. Zhan, Hydrophobic gold nanostructures via electrochemical deposition for sensitive SERS detection of persistent toxic substances, RSC Advances 5(18) (2015) 1344313450.

[33] A. Nsabimana, S.A. Kitte, T.H. Fereja, M.I. Halawa, W. Zhang, G. Xu, Recent developments in stripping analysis of trace metals, Current Opinion in Electrochemistry 17 (2019) 65-71.

[34] A. Wahl, K. Dawson, N. Sassiat, A.J. Quinn, A. O'Riordan, Nanomolar Trace Metal Analysis of Copper at Gold Microband Arrays, Journal of Physics: Conference Series 307 (2011) 012061. 
[35] J. Orozco, C. Fernández-Sánchez, C. Jiménez-Jorquera, Underpotential Deposition-Anodic Stripping Voltammetric Detection of Copper at Gold Nanoparticle-Modified Ultramicroelectrode Arrays, Environmental Science \& Technology 42(13) (2008) 4877-4882.

[36] N. Arjona, G. Trejo, J. Ledesma-García, L.G. Arriaga, M. Guerra-Balcázar, An electrokinetic-combined electrochemical study of the glucose electro-oxidation reaction: effect of gold surface energy, RSC Advances 6(19) (2016) 15630-15638.

[37] M. Pasta, F. La Mantia, Y. Cui, Mechanism of glucose electrochemical oxidation on gold surface, Electrochimica Acta 55(20) (2010) 5561-5568.

[38] Y. Xia, W. Huang, J. Zheng, Z. Niu, Z. Li, Nonenzymatic amperometric response of glucose on a nanoporous gold film electrode fabricated by a rapid and simple electrochemical method, Biosensors and Bioelectronics 26(8) (2011) 3555-3561.

[39] T.-M. Cheng, T.-K. Huang, H.-K. Lin, S.-P. Tung, Y.-L. Chen, C.-Y. Lee, H.-T. Chiu, (110)-Exposed Gold Nanocoral Electrode as Low Onset Potential Selective Glucose Sensor, ACS Applied Materials \& Interfaces 2(10) (2010) 2773-2780.

[40] C. Jeyabharathi, P. Ahrens, U. Hasse, F. Scholz, Identification of low-index crystal planes of polycrystalline gold on the basis of electrochemical oxide layer formation, Journal of Solid State Electrochemistry 20(11) (2016) 3025-3031. 